Methods FAP $\alpha$ expression in vivo was determined by immunohistochemistry in human synovial tissues of patients with RA and osteoarthritis (OA) as well as in hind paws of tumour necrosis factor-alpha transgenic (hTNFtg) mice, which develop a RA-like destructive arthritis. In vitro expression and regulation of FAP $\alpha$ was analysed in differentiated osteoclasts cocultured with osteoblasts, RA or OA synovial fibroblasts (SF) by PCR. To scrutinise the role of FAP $\alpha$ in osteoclastogenesis, we analysed the in vitro differentiation of osteoclasts from wildtype (WT) and FAP $/$ mice (Oncology Research, Boehringer Ingelheim RCV, Vienna) by TRAP staining. In order to assess the role of FAP in arthritis severity, we crossed FAP/ and hTNFtg mice and performed TRAP staining.

Results RA synovial tissues demonstrated a high expression of FAP $\alpha$ throughout the tissue whereas in OA samples FAP $\alpha$ was expressed only in the lining layer. In vitro, no expression of FAP $\alpha$ was found in differentiated preosteoclasts and osteoclasts, but coculture experiments showed that RASF, but not OASF or osteoblasts, induce the expression of FAP $\alpha$ in preosteoclasts and osteoclasts. Consistent with the selective induction of FAP $\alpha$ in osteoclasts by RASF, FAP $\alpha$ expression was detected in osteoclasts at the invasion front of the hyperplastic synovial tissues in joints of hTNFtg mice. FAP /- mice show a severely diminished osteoclast formation compared to WT. We also found a lesser amount of osteoclasts in the hind paws of FAP/hTNFtg compared to hTNFtg.

Conclusions The disease-dependent expression of FAP $\alpha$ by osteoclasts in human RA and hTNFtg mice suggests an important role of FAP $\alpha$ in joint destruction in RA. The selective induction of FAP $\alpha$ in preosteoclasts and osteoclasts by RASF indicate that FAP $\alpha$ may be regulated through the interaction with the pannus tissue. The fact, that under inflammatory conditions the loss of FAP led to a reduced number of osteoclasts in the hind paws and FAP deficient bone marrow derived macrophages showed a reduced osteoclast formation, suggest a role of this serine protease in macrophage/osteoclast precursor migration and differentiation.

\section{A8.6 FRZB IS A CRITICAL MODULATOR OF CANONICAL WNT SIGNALLING IN CARTILAGE BIOLOGY}

doi:10.1136/annrheumdis-2013-203222.6

S Thysen, F Cailotto, FP Luyten, R Lories. Laboratory of Tissue Homeostasis and Disease, Skeletal Biology and Engineering Research Center, Department of Development and Regeneration, KU Leuven, Belgium

Background and Objectives Polymorphisms in Frizzled-related protein $(F R Z B)$, a WNT antagonist, have been associated with osteoarthritis $(\mathrm{OA})$. However, a recent meta-analysis failed to find a consistent effect of FRZB genetic variants on OA susceptibility. Our transcriptomics analysis in $F R Z B^{-/}$mice provided evidence for a tight regulation of WNT signalling and highlighted the complex role for $F R Z B$ in joint homeostasis. We previously demonstrated that $F R Z B^{-1}$ mice have increased damage when dramatically challenged by papain, collagenase or severe inflammation. As these models are acute and short-term, we aimed to further investigate the effect of $F R Z B$ loss in a true translational model of $O A$ and to study molecular interactions in the ATDC5 micromass in vitro model using RT-PCR and Western blot analysis.

Materials and Methods Surgical destabilisation of the medial meniscus (DMM) was performed on the right knee of eight-week-old male $F R Z B^{-/}$and wild-type mice. Eight weeks after surgery, mice were sacrificed and histological scores were determined for the femoral and tibial articular surfaces following the OARSI histopathology initiative guidelines.

Results Overexpression of FRZB in ATDC5 micro-masses boosted chondrogenesis with up-regulation of Col2a1 and Aggrecan transcription, whereas downregulation of $F R Z B$ lead to a decreased expression of Colza1 and Aggrecan. These results corresponded with a reduction or increase in the activation of canonical WNT signalling pathway, respectively. Fluctuating levels of $F R Z B$ did not influence the Wnt/CamKII signalling pathway. The semi-quantitative $O A R S I$ score showed a significant increase in cartilage erosion in DMM-operated $F R Z B^{-1}$ mice compared to wild-type.

Conclusions Our data show that, in addition to the higher susceptibility to $\mathrm{OA}$ in acute induced models, $F R Z B^{-/}$mice are more prone to $O A$ in a full translational model of the disease characterised by slowly progressive joint damage. Overexpression of $F R Z B$ stimulates chondrogenesis by its inhibitory role on Wnt/beta-catenin signalling.

\section{A8.7 FUNCTIONAL IMPAIRMENT IN AN ANIMAL MODEL FOR RHEUMATOID ARTHRITIS ASSESSED AS CHANGES IN GAIT IS DUE TO JOINT DESTRUCTION BUT NOT SYNOVIAL INFLAMMATION PER SE}

doi:10.1136/annrheumdis-2013-203222.7

Gregor Bauer, Martin Willburger, Constantin Aschauer, Aurica Jelinek, Tetyana Shvets, Birgit Niederreiter, Josef Smolen, Kurt Redlich, Silvia Hayer. Medical University of Vienna, Department of Internal Medicine III, Division of Rheumatology, 1090 Vienna, Austria

Objective To investigate the individual impact of synovial inflammation, subchondral bone erosion or cartilage damage on functional impairment in an animal model of Rheumatoid Arthritis (RA).

Methods We analysed gait profiles in human tumour necrosis factor transgene (hTNFtg) animals, using the video-based Catwalk gait analysis system (from Noldus, Netherlands). In this system, mice run along an illuminated glass plate. A digital camera measures light emissions resulting from the contact of paws on the glass plate. We evaluated gait profiles at different time points of disease (6, 10, 15 week of age) in hTNFtg animals. Wildtype littermates served as controls. Bodyweight and clinical signs of arthritis including paw swelling and grip strength were also evaluated. To investigate whether gait changes are pain related, we treated hTNFtg animals with diclofenac (50 mg/kg, i.p.) at week 10 and week 15 after birth and analysed gait profiles before, as well as $1 \mathrm{~h}$ and $3 \mathrm{~h}$ after treatment. To investigate reversibility in impaired gait profils, we treated hTNFtg mice with anti-TNF (Infliximab $10 \mathrm{mg} / \mathrm{kg}$ body weight, $2 \times$ per week) for 5 weeks starting 6 and 10 weeks after birth. To analyse inflammatory joint destruction, we quantitatively assessed the extend of synovial inflammation, subchondral bone erosion and cartilage damage on hematoxylin and eosine (H\&E), tartrate-resistant acid phosphatase (TRAP) and toluidine-blue stained paw sections. We performed correlation studies between gait parameters and the histopathological components as well as clinical signs.

Results We identified several gait parameters among them weight bearing, stride length and contact area of the paw to be significantly decreased in hTNFtg animals compared to sex- and age-matched wildtype animals. Moreover, we found a marked reduction in maximum intensity, an indicator for weight bearing, in week 10 and 15 compared to week 6 old hTNFtg mice. Similar effects were seen in print width, print area, print length, max contact max intensity and max contact area at different stages of disease. Interestingly, analgesic treatment with diclofenac $(50 \mathrm{mg} / \mathrm{kg}$, i.p. $)$, resulted in a better improvement of weight-bearing parameters in 10 week old hTNFtg mice than in 15 week old hTNFtg animals indicating an pain independent, irreversible functional impairment in progressed disease. To further investigate to which extend synovial inflammation, subchondral bone erosion or cartilage damage are responsible for the functional impairment of joints, we correlated these components with changes in different gait parameters. We observed strong correlations of various gait parameters with the amount of cartilage damage, whereas subchondral bone erosions correlated to a lesser extend and synovial inflammation did not correlate at all. 
In addition, anti-TNF treatment starting at early stage (6 weeks) or progressed disease (10 weeks) markedly improved clinical as well as histopathological signs and reversed gait changes.

Conclusions Video-based Catwalk gait analysis is a useful tool for quantitative assessment of functional impairment in inflammatory, destructive arthritis. Joint destruction due to cartilage damage but not synovial inflammation per se is the most important component leading to functional impairment of hTNFtg mice.

\section{A8.8 HYDROGEN SULPHIDE INHIBITS IL-1 $\beta$ STIMULATION OF FIBROBLAST-LIKE SYNOVIOCYTES FROM OSTEOARTHRITIS PATIENTS IN A 3-D MODEL}

doi:10.1136/annrheumdis-2013-203222.8

'D Sieghart, ${ }^{2 H P}$ Kiener, ${ }^{1}, 2 \mathrm{G}$ Steiner, 'B Kloesch. ' $L$ udwig Boltzmann Cluster Rheumatology, Balneology and Rehabilitation, Ludwig Boltzmann Institute of Rheumatology and Balneology, Vienna, Austria; ' $M e d i c a l$ University of Vienna, Department of Internal Medicine III, Division of Rheumatology, Vienna, Austria

Objective Osteoarthritis $(\mathrm{OA})$ is a degenerative and most common joint disease which shows characteristic features like loss of cartilage, formation of osteophytes and alteration of subchondral bone leading to joint impairment and pain. The pathogenesis of $\mathrm{OA}$ is still not fully understood. Fibroblast-like synoviocytes (FLS), occurring in the intimal lining layer of the synovial membrane, were shown to promote secondary synovitis by the release of proinflammatory cytokines and matrix-metalloproteinases (MMPs). The objective of this study was to analyse the possible antiinflammatory potential of hydrogen sulphide $\left(\mathrm{H}_{2} \mathrm{~S}\right)$ on activated FLS cultured in three-dimensional micromass culture.

Methods Primary cell lines based on FLS derived from patients with OA were cultivated in spherical extracellular matrix micromasses. Micromass cultures were stimulated for $1 \mathrm{~h}$ with IL-1 $\beta$ $(10 \mathrm{ng} / \mathrm{ml})$ only or with IL-1 $\beta$ plus either $0.125 \mathrm{mM}$ or $1 \mathrm{mM}$ of sodium hydrogen sulphide ( $\mathrm{NaHS}$ ). As a control, cultures were treated with PBS only. Treatments were applied on day three, five, seven, nine, 12, 14, 16 and 19. Micromasses were cultured for 21 days, fixed with paraformaldehyde, sectioned and stained for hematoxylin and eosin (H\&E), IL-6 or MMP-3. Secretion of IL-6 was analysed by enzyme-linked immunosorbent assay (ELISA).

Results We observed the spontaneous formation of a compacted, lining layer-like architecture by OA-FLS, already described for rheumatoid arthritis (RA)-FLS. Untreated cultures, in addition, showed clusters of elongated cells underneath the condensed cell layer.

A cellular response, which included increased formation of synovial lining as well as changes in cell morphology, could be seen after stimulation with the pro-inflammatory cytokine IL-1 $\beta$. Treatment with $1 \mathrm{mM}$ of $\mathrm{NaHS}$ had the potential to inhibit structural changes caused by cell activation induced by IL-1 $\beta$. Furthermore, $\mathrm{H}_{2} \mathrm{~S}$ treatment reduced the IL-1 $\beta$ stimulation-related elevated levels of IL- 6 secretion.

Conclusions The ability of NaHS to inhibit the development of cellular responses to pro-inflammatory IL- $1 \beta$ could be considered a cartilage protective effect and has to be elucidated in more detail.

\section{A8.9 CHARACTERISATION OF CELL METABOLIC CHANGES IN OSTEOARTHRITIC LABRUM CELLS}

\section{doi:10.1136/annrheumdis-2013-203222.9}

'Juchtmans Nele, AM Dhollander Aad, 'Lambrecht Stijn, 'Elewaut Dirk. Department of Rheumatology, Ghent University, Ghent, Belgium

Background and Objectives Little is known about the cell metabolism of the acetabular labrum, a horseshoe-shaped structure sealing the hip joint. It performs similar functions as the menisci in the knee though the metabolic aspects of labrum cells show fibrochondrocyte characteristics, different from menisci and cartilage. The goal of this study was to investigate whether labrum cells derived from osteoarthritic tissue have different cell metabolic characteristics compared with labrum cells derived from healthy tissue. Furthermore this study aimed to investigate specific processes of functional importance in the pathology of osteoarthritis.

Materials and Methods Labrum cells from 5 OA patients en 3 healthy control patients were isolated and cultured in the 3-Dimensional alginate culture system. A genome wide gene expression analysis was performed using the Affymetrix microarray technology. Differential gene expression levels were confirmed on additional patient samples by quantitative PCR (qPCR), western blot and immunohistochemistry.

Results Gene expression analysis showed, as expected, downregulation of aggrecan and several matrix degrading enzymes such as (MMP's) and (ADAMTS's) were up regulated in OA. qPCR confirmation occurred for 20 of the most pronounced differences between normal and OA labrum. Of these 20 genes the Short leucine-rich repeat proteins (SLRP's) asporin (ASPN) and osteomodulin (OMD) were of special interest, both showed a reduced expression level and protein presence in $\mathrm{OA}$ labrum cells. More over stimulation in vitro of labrum cell with IL1 $\beta$ down regulated the expression of both proteins.

Conclusions Labrum cells derived from OA patients show a consistent altered metabolism compared to cells isolated from healthy controls. The reduced expression of ECM genes in OA labrum point towards a distorted ECM synthesis. Together with the observed increase in ECM degradation by MMP's, our data indicate that both distorted anabolism and catabolism contribute to degeneration of labrum tissue in OA. The short leucine-rich proteins (SLPR's) ASPN and $\mathrm{OMD}$ were of special interest as these exhibited the most pronounced expression differences. In conclusion this study clearly indicates that similar events occur during OA development in labrum and cartilage. Though labrum specific expression differences are observed that merit further investigation.

\section{A8.10 MAPK-ACTIVATED PROTEIN KINASE-3 (MK3) REGULATES OSTEOCLAST DIFFERENTIATION AND BONE HOMEOSTASIS}

doi:10.1136/annrheumdis-2013-203222.10

${ }^{1}$ Tobias Braun, 'Gisela Ruiz Heiland, 'Georg Schett, ${ }^{1,2}$ Jochen Zwerina. 'Department of Medicine 3, University of Erlangen-Nuremberg, Erlangen, Germany; ${ }^{2}$ Ludwig Bolzmann Institut of Osteology at the $4^{\text {th }}$ medical Department, Hanusch Hostpital, Vienna, Austria

Background and Objectives MAPK-activated protein kinase-2 (MK2) plays a key role in bone homeostasis. MK2 deficient mice have increased bone mineral density associated with reduced number of osteoclasts. Osteoclast differentiation is impaired due to reduced DNA binding activity of the transcription-factors c-fos and NFATc1. MK3 is besides MK2 an important downstream target of MAPK p38. MK3 plays together with MK2 an important role in regulation of cytokine secretion and inflammation. Aim of our study is to determine the role of $\mathrm{MK} 3$ and $\mathrm{MK} 3 / \mathrm{MK} 2$ interaction in bone homeostasis.

Materials and Methods We analysed trabecular bone structure of the tibia of 12 week old wild type, MK3 deficient, MK2 deficient and $M K 2 / 3$ deficient mice by micro CT. Tibiae were decalcified and paraffin sections were stained with tartrate-resistant acid phosphatase (TRAP) to determine osteoclast number/bone perimeter and osteoclast surface/bone surface by histomorphometry. Bone marrow cells of wild type, MK3 deficient, MK2 deficient and MK2/3 deficient mice were simulated with M-CSF and RANKL and stained for TRAP after four days to investigate osteoclast differentiation ex vivo.

Results Analysis of trabecular bone structure showed increased trabecular volume, increased trabecular number and decreased 